\title{
A Marketing Analysis of Herbal Anti-Inflammatory Drugs
}

\author{
Aleshnikova K.Yu. ${ }^{1,{ }^{*}}$ Gribkova E.I. ${ }^{1}$ Dzhavakhyan M.A. ${ }^{2}$ \\ ${ }^{1}$ Peoples' Friendship University of Russia, Moscow, Russia \\ ${ }^{2}$ FGBNU «VILAR», Moscow, Russia \\ *Corresponding author.Email: k.aleshnikova@yandex.ru
}

\begin{abstract}
The main purpose of this study is to conduct a marketing analysis of anti-inflammatory drugs (AID). Due to the fact that this analysis was aimed at identifying anti-inflammatory drugs of plant origin in the form of medical pencils, positioning of this dosage form was carried out. The study included three stages. The first stage involved the analysis of the main assortment characteristics of anti-inflammatory drugs, which made it possible to reveal anti-inflammatory drugs with eucalyminum that were not registered in the Russian pharmaceutical market. At the second stage, a patent search was carried out. It confirmed the results of the first stage. At the final stage, using a sociological survey, the positioning of the new dosage form was carried out. The study took place in medical organizations. 102 general practitioners took part in the survey and interviewing. All profiles were found to be valid. The following results were obtained: when assessing the degree of severity and significance, doctors chose the following criteria: efficacy, safety (side effects), speed of the therapeutic effect and possibility of taking the drug by special groups of consumers. When determining these criteria in terms of significance, doctors prioritized efficacy and safety, as well as speed of the therapeutic effect and its duration.
\end{abstract}

Keywords: assortment analysis, anti-inflammatory effect, positioning, medicinal product, severity, importance, level of significance

\section{INTRODUCTION}

When developing a new drug, it is necessary to determine its place on the pharmaceutical market. An analysis of the drugs market is required. An assortment analysis allows you to identify dosage forms that are least common in the market. When developing a medicinal product of plant origin, plants that have been already used were identified. Patent searches are carried out for similar purposes. This analysis allows you to determine dosage forms, composition, therapeutic and effects of the drugs. All properties of the new drug must meet the needs of end and intermediate consumers (doctors). Positioning is carried out and competitive advantages of the drug are determined, which will allow us to improve the competitiveness of the new drug. Currently, the development of medicinal products of plant origin is a promising direction, despite the fact that they are present in large quantities in the Russian pharmaceutical market. These drugs are in demand. The medicinal pencils are used when it is required to exert the effect of a medicinal substance on a small area of skin or mucous membrane where the inflammatory process is observed [1-3].

\section{METHODS AND MATERIALS}

The marketing analysis of anti-inflammatory drugs involved:

- development of a database for the - drugs;

- $\quad$ analysis of the main assortment characteristics;

- $\quad$ patent search for anti-inflammatory drugs;

- $\quad$ positioning of the new dosage form - medicinal pencils with eucalymin.

The database of approved anti-inflammatory drugs was compiled using the content analysis. The materials used were: the state register of medicines, the state register of instructions for the medical use of drugs. The patent search was carried out on the basis of the Internet - the Patent Office of the Russian Federation. As a result of the content analysis, we selected 100 patents for the drugs with antimicrobial, wound healing and anti-inflammatory effects. The time period was 27 years. The patents were studied and analyzed. During positioning, 102 surveys of general practitioners were conducted. The representativeness of the sample was determined by the formula of irreversible random selection. 
The following research methods were used: content analysis, questioning, interviewing, statistical, mathematical, analysis and graphic analyses.

\section{RESULTS}

The marketing analysis of anti-inflammatory drugs included several stages. At the first stage, an expanded database of anti-inflammatory drugs was compiled. To compile it, we used the State Register of Medicines and, instructions for medical use. At the next stage, the main assortment characteristics of the drug group were studied. The analysis was carried out: by manufacturers, by dosage forms, by pharmacotherapeutic effects, etc. At the third stage, we carried out a patent search using the database of the Patent Office of the Russian Federation. As a result, 100 patents were selected. This stage was aimed at identifying the dosage form of anti-inflammatory medicinal pencils. At the final stage, we positioned the dosage form of medicinal pencils in terms of severity and significance. Original questionnaires were developed and a sociological survey of intermediate consumers was conducted. The respondents were general practitioners. The assessment was carried out according to the following characteristics:

1. Efficiency

2. Effect force

3. Safety (side effects).

4. Cost per dose.

5. Speed of the therapeutic effect.

6. Duration of the therapeutic effect.

7. Variability of the dosage regimen

8. Prevention effect

9. Additional indications.

10. Use by special groups of patients (children, geriatric patients).

At the first preparatory stage of the study, with the help of official reference books, we compiled an expanded database on anti-inflammatory drugs. All information was summarized in one table, according to which, at the second stage of the study, the anti-inflammatory drugs were analyzed. The database included:

- trade and international non-proprietary name;

- company and country of origin;

- dosage form;

- ATC classification code;

- date of registration;

- active substance;

- cost;

- taking by a special category of patients (children, geriatric patients);

- $\quad$ side effects;

- pharmacotherapeutic effect;

- name of the plant organ used in the production of drugs.

An analysis of the database showed that 568 drugs were registered in the Russian pharmaceutical market.
They possess both an anti-inflammatory effect in combination with other effects, and an anti-inflammatory mono-effect (Fig. 1): antiseptic and anti-inflammatory agent $-0.5 \%$; antiseptic and anti-inflammatory agent of plant origin $-28 \%$; anti-inflammatory agent $-6.2 \%$; antiinflammatory agent for topical application - $30 \%$; antiinflammatory agent for external use $-0.8 \%$; antiinflammatory agent of herbal origin for topical application $-0.5 \%$; anti-inflammatory agent of herbal origin $-32 \%$; anti-inflammatory, antibacterial, antifungal agent $-0.5 \%$; anti-inflammatory analgesic antiseptic local agent $-1 \%$; wound healing and anti-inflammatory agent $-0.5 \%$.

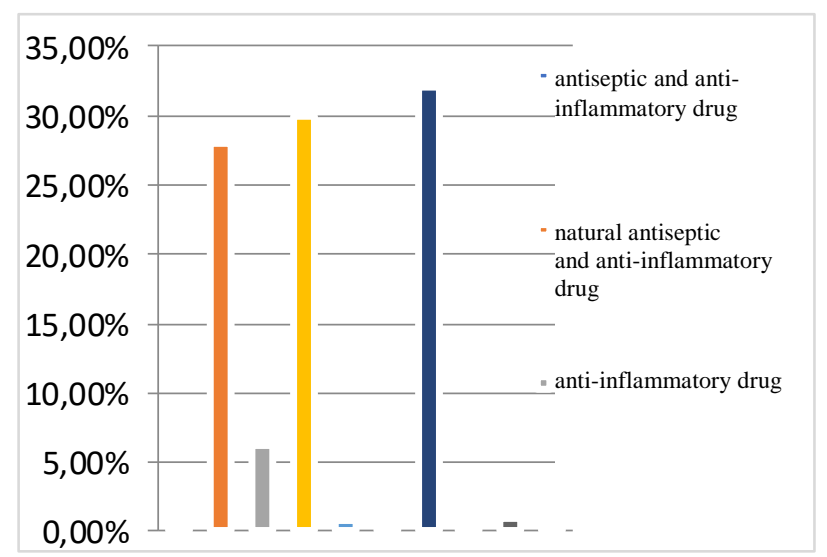

Figure 1 Distribution of drugs by their therapeutic effect

Due to the fact that the purpose of this analysis is to identify anti-inflammatory drugs of herbal origin and herbal medicinal preparations with the same effect, a further analysis was carried out in two directions. As a result, it was revealed that the majority of domestic $(57 \%)$, and foreign drugs (43\%) were registered. Among the countries that produce anti-inflammatory drugs of herbal origin are: Bulgaria, Germany, Denmark, Israel, India, Spain, Cyprus, China, Cuba, the Netherlands, Poland, Belarus, Romania, Slovenia, Ukraine. Next, we carried out an analysis of plants from which medicinal anti-inflammatory drugs of herbal origin are produced. For the production of these medicinal products and herbal medicinal preparations, the following plants were used: chamomile and calendula officinalis. The following types were used as raw materials: chamomile flowers and calendula medicinal flowers. In terms of composition, the Russian pharmaceutical market offers single-component drugs $-68 \%$ and multicomponent drugs $-32 \%$. The following combinations of medicinal plant materials were identified: calendula + chamomile + yarrow (LP-Rotokan, Zhekaton); marsh calamus rhizomes + arnica herb + oak bark + peppermint leaves + chamomile flowers + common thyme herb + medicinal sage leaves (LP-Stomatofit), etc.

In terms of dosage forms, soft dosage forms have a clear advantage. Among them, ointments make up $21 \%$; among solid dosage forms, tablets make up $31 \%$; liquid dosage forms are represented by infusions (13\%) (Fig. 2).

We also identified herbal medicinal preparations with an anti-inflammatory effect. For their production, 
manufacturers use such morphological plant organs as flowers, grass, leaves.

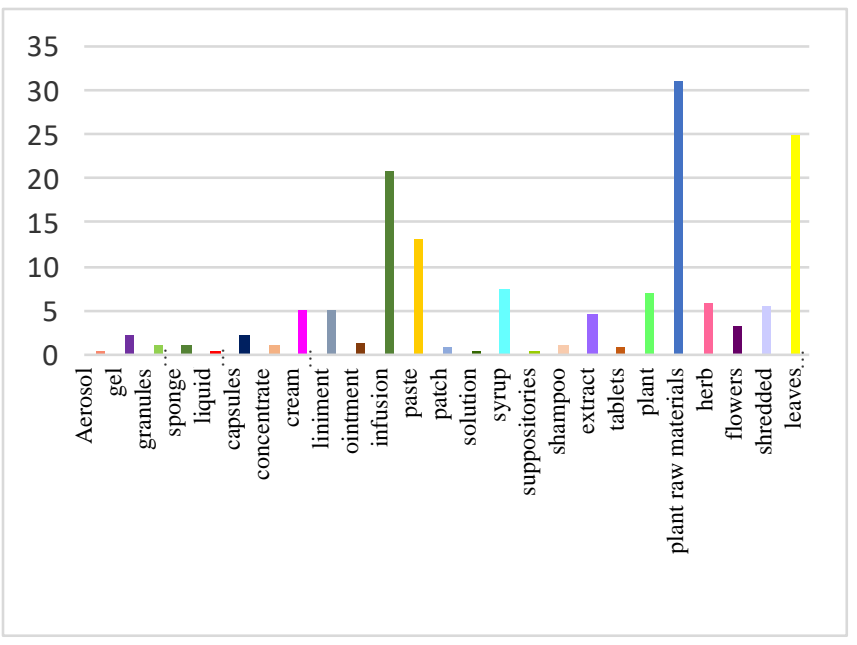

Figure 2 Analysis of dosage forms of antiinflammatory drugs

At the second stage, we analyzed patents for new drugs, which had at least one of the following pharmacological effects: antimicrobial, wound healing and anti-inflammatory, due to the fact that eucalymin has the above effects. An analysis of the patents showed that the largest number of patents were granted in 1997, 2008 and 2012. An analysis of patents for registered dosage forms, as well as an analysis of the range of approved medicinal products showed that there were no medicinal antiinflammatory pencils. The patents for the following dosage forms have been found: ointment, gel, cream, liniment, aerosol, capsules, tincture, suppositories, balm. Ointments, suppositories and gels prevailed. The analysis of registered patents revealed five positions of cosmetic products and three medicines for animals. No dosage form of anti-inflammatory drugs has been identified, which makes it possible to develop this dosage form with eucalymin.
At the final stage, we positioned anti-inflammatory medicinal pencils. We have developed an original questionnaire. The respondents were general practitioners. To calculate the representative sample, the formula of irreversible random sampling was used (1)

$$
n=\frac{t^{2} \sigma}{\Delta^{2}}
$$

where $n$ - minimum sample size; $t$ - Student's confidence factor; $\sigma$-dispersion; $\Delta$ - marginal error.

The degree of research accuracy was 0.95 (accordingly, the Student's coefficient of reliability was 1.96), the marginal sampling error was $0.1(10 \%)$. Under these conditions, the minimum sample size was96.04. Accordingly, the sample size should be at least 97 observation units. As part of our research, 102 specialists took part in the survey, thereby ensuring the representativeness of the research. [4-6].

Doctors were to evaluate a new dosage form of medicinal pencils with eucalymin, which has an antiinflammatory effect. The assessment was carried out according to two indicators - severity and significance of the following characteristics: efficacy, effect, safety (side effects), cost of per dose, the rate of onset of the therapeutic effect, duration of the therapeutic action, variability of the dosage regimen, possibility of taking for prophylaxis, additional indications, possibility of using the drug by special groups of patients (children, geriatric patients). When displaying the results, the upper and lower bounds for the average sum of scores were set and the following indicators were determined: very high, key, very significant, significant, high, medium, secondary, low, very low. The indicators were ranked from the most significant to the least significant ones. As a result, the following results were obtained (table 1). As can be seen from the tabular data, the highest scores were given to efficacy and safety; speed of the therapeutic effect and possibility of taking the drug by special groups were also significant indicators.

Table 1 Results of assessment of drugs by doctors according to the criteria for choosing a new drug

\begin{tabular}{|l|l|l|}
\hline \multicolumn{2}{|c|}{ Drug selection criteria } & \multicolumn{2}{c|}{ Interpretation of scale levels } \\
\cline { 2 - 3 } & \multicolumn{1}{c|}{ Mignificance } & Manifestation \\
\hline Efficiency & Key parameter & high \\
\hline Safety (side effects) & Very significant & medium \\
\hline Drug cost per dose & significant & high \\
\hline Rate of the onset of the therapeutic effect & Very significant & medium \\
\hline Duration of the therapeutic effect & significant & medium \\
\hline Dosing regimen variability & significant & high \\
\hline $\begin{array}{l}\text { Possibility of use by special groups of patients (children, } \\
\text { geriatric patients, etc.) }\end{array}$ & high & low \\
\hline Prevention effect & significant & Very low \\
\hline Additional indications & insignificant & \\
\hline
\end{tabular}


Next, we calculated the relative level of significance of each criterion. The graph was constructed based on the results of a survey (Figure 3) The calculation of the relative level of significance of each criterion was carried out according to formula (2):

Significance level $=\mathrm{a} / \mathrm{A} \times 100 \%,(2)$

where $\mathrm{A}$ - selection criterion score; $\mathrm{A}$ - the sum of scores of the criterion with the highest value.

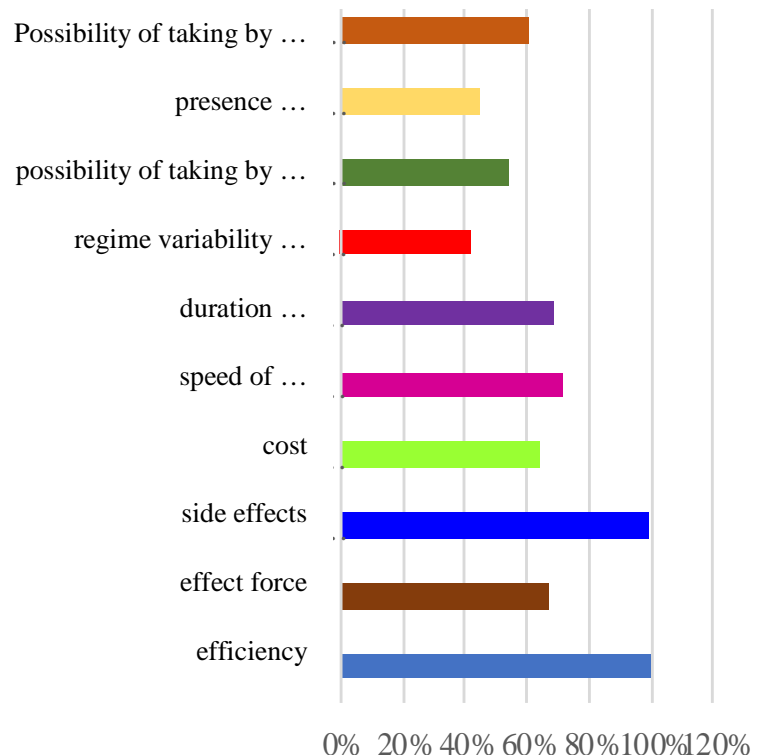

Figure 3 Significance level of positioning criteria when interviewing doctors

Questionnaires for doctors also contained questions about the need to develop a new anti-inflammatory drug based on eucalymina. According to the survey, the overwhelming majority of specialists spoke in favor of the need to create drugs of this group. The following advantages of the developed dosage form in comparison with ointments were observed: the possibility of using medicinal substances with different physicochemical properties, convenience and economy, compactness, hygienic packaging, portability, low likelihood of infection on the damaged skin area. [7-10].

\section{CONCLUSION}

Using the developed database, an analysis of the assortment of anti-inflammatory drugs of plant origin by the country of origin, dosage forms, therapeutic groups, etc. was carried out. This analysis made it possible to determine that anti-inflammatory drugs in the form of pencils are absent in the Russian pharmaceutical market. The patent search confirmed these findings. The survey identified the characteristics of drugs which have to be taken into account when developing an advertising campaign, thereby improving the competitiveness of a new dosage form of medical pencils with eucalymin, which have anti-inflammatory effects. Thus, the development of the composition and technology of anti-inflammatory medicinal pencils containing eucalymin is relevant.

\section{REFERENCES}

[1] A.V. Zozulev, Consumer Behavior, Textbook, Knowledge, Kiev, 2004, 364 p.

[2] A.V. Korotkov, Marketing research: Textbook for universities, UNITY-DANA, Moscow, 2005, 304 p.

[3] V.V. Dorofeeva, V.D. Chan, Exploring the Factors Affecting Pharmaceutical Consulting in Vietnamese Pharmacies, Remedium, Magazine about the drug and med. equipment market 4 (2019) 32-35.

[4] E.G. Elyashevich, New functions of a pharmacist at the present stage of development of pharmacy, Vest. of VSMU 14(1) (2015) 6-11.

[5] I.V. Kosova, E.E. Loskutova, T.P. Lagutkina, V.V. Dorofeeva, A.A. Teodorovich, Economics and organization of pharmacy: textbook for student institutions of vocational education, ed. by I.V. Kosova, Publ. Center "Academy", Moscow, 2018, 448 p.

[6] N.B. Dremova, N.I. Pankova, T.G. Afanasyeva, Otseka positioning of pharmaceutical products: theoretical foundations and practical application, Monograph, Antares, Voronezh, 2010, 176 p.

[7] K.Yu. Aleshnikova, M.A. Javakhyan, E.I. Gribkova, Comparative analysis of the pharmaceutical market of medical pencils with venotonic, antiviral and antimicrobial action, Innovations in the health of the nation, Collection of materials of the IV All-Russian scientific and practical conference with international participation, 2016, pp. 224-228.

[8] K.Yu. Aleshnikova, M.A. Javakhyan, Technological aspects of the development of medicinal pencils with eucalymin, Quest. of biolog., med. and pharmac. chem. 22(3) (2019) 37-41. ISSN 1560-9596.

[9] E.M. Abrams, M. Ben-Shoshan, Delabeling penicillin allergy: Is skin testing required at all? J. Allergy Clin. Immunol. Pract. 7(4) (Apr 2019) 1377.

[10] Ya.I. Khazhdai et al., Features of studying the harmlessness of ointments and suppositories, Pharmacy 32(1) (1983) 22-26. 